\title{
ANALYSIS OF A CLASS OF PROBABILITY PRESERVING MEASURE ALGEBRAS ON COMPACT INTERVALS
}

\author{
WILLIAM C. CONNETT AND ALAN L. SCHWARTZ
}

\begin{abstract}
The measure algebras of the title are those which are also hypergroups with some regularity conditions. Examples include the convolutions associated with Jacobi polynomial series and Fourier Bessel series. It is shown here that there is a one-to-one correspondence between these hypergroups and a class of Sturm-Liouville problems which have the characters of the hypergroup as eigenfunctions. The interplay between these two characterizations allows a detailed analysis which includes a Hilb-type formula for the characters and asymptotic estimates for the Plancherel measure and the eigenvalues of the associated Sturm-Liouville problem.
\end{abstract}

\section{INTRODUCTION.}

In the last twenty years, a number of concrete convolution measure algebras have been studied in great detail, and new structures continually emerge (see $[2,3,4,5,9,15]$, and the references cited in those articles). Many of these are probability-preserving algebras of measures on an interval; indeed, many are examples of the type II one-dimensional hypergroups discussed in [17]. When the interval is compact, these examples share many properties of the convolution structure associated with Jacobi polynomials described in [9] and below. In an effort to understand the general behavior of such measure algebras, we introduce the notion of Jacobi type hypergroups, a general class which includes many examples. This article is devoted to the description of this class and its properties.

\subsection{Examples.}

A. Jacobi and ultraspherical convolution. Let $\alpha, \beta>-1$ then the system of polynomials $\left\{P_{n}^{(\alpha, \beta)}\right\}$ orthogonal on $H=[-1,1]$ with respect to the measure $d v(s)=(1-s)^{\alpha}(1+s)^{\beta} d s$ and normalized by $P_{n}^{(\alpha, \beta)}(1)=\left(\begin{array}{c}n+\alpha \\ n\end{array}\right)$ are called the Jacobi polynomials of order $(\alpha, \beta)$; the ultraspherical polynomials of order $\lambda$ are obtained by setting $\alpha=\beta=\lambda-\frac{1}{2}$ and using a different normalization. The

Received by the editors May 3, 1988 and, in revised form, September 27, 1988.

1980 Mathematics Subject Classification (1985 Revision). Primary 43A10; Secondary 34B25, $42 \mathrm{C} 05$.

Key words and phrases. Measure algebra, hypergroup, Sturm-Liouville problems.

The second author wishes to express his gratitude to the School of Mathematical Sciences at Tel-Aviv University for its hospitality during the preparation of this paper. 
standard reference on the subject is Szegö [19]; some additional properties and references are also found in [3].

Gasper [9] showed that if $\alpha \geq \beta$ and either $\beta \geq-\frac{1}{2}$ or $\alpha+\beta \geq 0$, then for each $s, t \in H$, there is a nonnegative measure $\sigma_{s t} \in M(H)$ such that if $R_{n}(s)=\left(\begin{array}{c}n+\alpha \\ n\end{array}\right)^{-1} P_{n}^{(\alpha, \beta)}(s)$ then

$$
\int_{H} R_{n}(u) d \sigma_{s t}(u)=R_{n}(s) R_{n}(t) .
$$

A Banach algebra $(H, * ; \alpha, \beta)$ is obtained if for $\mu, \nu \in M(H), \mu * \nu$ is defined by

$$
\int_{H} f(r) d(\mu * \nu)(r)=\int_{H} \int_{H} \int_{H} f(r) d \sigma_{s t}(r) d \mu(s) d \nu(t)
$$

for all $f \in C(H)$ (cf. [12, Lemma 3.1.C]). This example will be continued in $\S \S 1.4$ and 5 .

B. Fourier-Bessel series. Let $j_{0}, j_{1}, j_{2}, \ldots$ be the positive zeros of $J_{\alpha}(x)$, the Bessel function of the first kind of order $\alpha$, and define

$$
\begin{gathered}
\mathscr{J}_{\alpha}(x)=2^{\alpha} \Gamma(\alpha+1) x^{-\alpha} J_{\alpha}(x), \\
\widetilde{J}_{k}^{\alpha}(x)=\mathscr{J}_{\alpha}\left(j_{k} x\right) / \mathscr{J}_{\alpha}\left(j_{0} x\right),
\end{gathered}
$$

and

$$
\tilde{w}(x)=\left[\mathscr{J}_{\alpha}\left(j_{0} x\right)\right]^{2} x^{2 \alpha+1} .
$$

Markett [15] has shown that if $\alpha \in\left\{\frac{1}{2}, \frac{3}{2}, \frac{5}{2}\right\}$ then there is a nonnegative function $\widetilde{K}_{F B}^{\alpha}(\xi, x, y)$ on $H=[0,1]$ such that

$$
\int_{0}^{1} \widetilde{J}_{k}^{\alpha}(\xi) \widetilde{K}_{F B}^{\alpha}(\xi, x, y) \tilde{w}(\xi) d \xi=\widetilde{J}_{k}^{\alpha}(x) \widetilde{J}_{k}^{\alpha}(y) \quad(0<x, y<1) .
$$

This gives rise to a convolution on $M(H)$ by defining

$$
\int_{H} f(\xi) d(\mu * \nu)(\xi)=\int_{H} \int_{H} \int_{H} f(\xi) \widetilde{K}_{F B}^{\alpha}(\xi, x, y) \tilde{w}(\xi) d \xi d \mu(x) d \nu(y) .
$$

C. Perturbed ultraspherical series. Consider the linear differential operator

$$
L^{\alpha} y=y^{\prime \prime}+(2 \alpha+1) \cot \theta y^{\prime} \quad(0<\theta<\pi)
$$

which has an eigenfunctions $P_{n}^{(\alpha+1 / 2)}(\cos \theta)$ where $P_{n}^{(\alpha+1 / 2)}$ is the ultraspherical polynomial of order $\alpha+\frac{1}{2}$ and degree $n$. Let $q$ be a continuous function on $(0, \pi)$ such that $\theta q(\theta)$ is real-analytic at zero, $q(\theta)$ is nonincreasing in $(0, \pi / 2)$, and $q(\pi-\theta)=q(\theta)$. Define $L_{q}^{\alpha} y=\left(L^{\alpha}-q\right) y ;$ C. Markett and the authors showed in [2] that the eigenfunctions of this operator satisfy a product formula analogous to equation (1.3) with a nonnegative kernel, so it is possible to define a convolution by means of a formula analogous to equation (1.4). 
1.3. Hypergroups. The structures described in the preceding section are examples of hypergroups which will now be defined.

Let $H$ be a locally compact space and let $M(H)$ denote the bounded Borel measures on $H$; if $\mu \in M(H), \operatorname{supp}(\mu)$ is the support of $\mu$. The unit point mass concentrated at $s$ is indicated by $\delta_{s} ; C(H)$ is the space of continuous complex-valued functions on $H$; and $C_{c}(H)$ consists of all $f$ in $C(H)$ with compact support.

If $M(H)$ is a Banach algebra with multiplication * (called a convolution), then $(H, *)$ is a hypergroup if the following axioms are satisfied:

H1. If $\mu$ and $\nu$ are probability measures, then so is $\mu * \nu$.

H2. The mapping $(\mu, \nu) \rightarrow \mu * \nu$ is continuous from $M(H) \times M(H)$ into $M(H)$ where $M(H)$ is given the weak topology with respect to $C_{c}(H)$.

H3. There is an element $e \in H$ such that $\delta_{e} * \mu=\mu * \delta_{e}=\mu$ for every $\mu \in M(H)$.

H4. There is a homeomorphic mapping $s \rightarrow s^{\vee}$ of $H$ into itself such that $s^{\vee \vee}=s$ and $e \in \operatorname{supp}\left(\delta_{s} * \delta_{t}\right)$ if and only if $t=s^{\vee}$.

H5. For $\mu, \nu \in M(H)(\mu * \nu)^{\vee}=\nu^{\vee} * \mu^{\vee}$ where $\mu^{\vee}$ is defined by

$$
\int_{H} f(s) d \mu^{\vee}(s)=\int_{H} f\left(s^{\vee}\right) d \mu(s) .
$$

H6. The mapping $(s, t) \rightarrow \operatorname{supp}\left(\delta_{s} * \delta_{t}\right)$ is continuous from $H \times H$ into the space of compact subsets of $H$ as topologized in [16].

More complete discussions of hypergroups can be found in $[8,12]$ and the surveys [11 and 14].

This paper continues the program begun in [17] to classify one-dimensional hypergroups. Our interest here is limited to hypergroups $(H, *)$ where $H$ is a compact interval. The inspiration for the classification is the family of hypergroups $(H, * ; \alpha, \beta)$ of Example A. Each of these is a member of the class we will call Jacobi type $(\alpha, \beta)$ hypergroups.

The definition of this class depends on the moments $M_{1}$ and $M_{2}$ defined below. These are intrinsic to the hypergroup and they provide for the classification in a fairly direct way; with the aid of the moments we can show (Theorems 2.1 and 2.2) that every hypergroup defined on a compact interval which satisfies certain regularity conditions is included in our classification.

In $\S 1.4$ we show that the hypergroups $(H, * ; \alpha, \beta)$ are indeed of Jacobi type $(\alpha, \beta)$, but the computation, even in this concrete case, is difficult, so that it is desirable to have a simpler means of achieving the classification. The clue is given by the fact that each polynomial $R_{n}$ defines a homomorphism on $(H, * ; \alpha, \beta)$ by $\mu \rightarrow \int_{H} R_{n} d \mu$ (cf. equations (1.1) and (1.2)), and that the $R_{n}$ 's are the eigenfunctions of a Sturm-Liouville problem. This is the viewpoint adopted and generalized in [6 and 7]. The parameters $\alpha$ and $\beta$ are readily extracted from the differential operator of the Sturm-Liouville problem.

This idea generalizes. Indeed, a unique Sturm-Liouville problem of a certain canonical type is associated with each Jacobi type $(\alpha, \beta)$ hypergroup, the pa- 
rameters can be extracted from the differential operator, and the characters form a complete set of eigenfunctions; this is proved in Theorem 3.6. Thus the classification can be achieved by simply examining the canonical Sturm-Liouville operator.

Since many examples, in particular B and C, are defined by constructing a hypergroup with characters that are known to be eigenfunctions, this theorem becomes the more useful tool for classification.

In $\S 4$, the classification and the associated Sturm-Liouville problem are used to obtain detailed information about some of the objects required for harmonic analysis of Jacobi type $(\alpha, \beta)$ hypergroups. In particular, asymptotic estimates for the characters analogous to the classic Hilb-type formulas are obtained in Theorem 4.1. An explicit formula for the Haar measure is obtained in Theorem 4.4. Theorem 4.6 contains precise estimates for the eigenvalues of the associated problem and an asymptotic estimate of the Plancherel measure. That the linear combinations of characters are dense in $C[0, \pi]$ is shown in Theorem 4.7.

In a future paper, we will show that these properties are among those essential for detailed analysis of the hypergroups. In particular, we shall present an analogue of the Hardy-Littlewood Maximal Theorem. ${ }^{1}$

The hypergroup $(H, *)$ is differentiable if whenever $f \in C_{c}\left(H^{\circ}\right)$, the continuous functions on $H$ with compact support in the interior of $H$, is $p$ times continuously differentiable, then $u(s, t)=\int_{H} f(r) d\left(\delta_{s} * \delta_{t}\right)(r)$ is $p$ times continuously differentiable on the interior of $H \times H$. For $\mu=1,2$ let $k_{\mu}$ be the largest positive integer $k$ such that for every $t$ interior to $H$

$$
M_{\mu}(s, t)=\int_{H}(r-t)^{\mu} d\left(\delta_{s} * \delta_{t}\right)(r)=O\left((s-e)^{k}\right)
$$

(the bound may depend upon $t$ ). If equation (1.5) is satisfied for no positive integer $k$, let $k_{\mu}=\infty$. Similarly define $\tilde{k}_{\mu}$ as the largest integer such that

$$
\widetilde{M}_{\mu}(s, t)=\int_{H}(r-s)^{\mu} d\left(\delta_{s} * \delta_{t}\right)(r)=O\left((t-e)^{k}\right) .
$$

The quantities $M_{\mu}(s, t)$ and $\widetilde{M}_{\mu}(s, t)$ are called moments. $(H, *)$ is regular if it is differentiable and at least one of $k_{1}, \tilde{k}_{1}, k_{2}, \tilde{k}_{2}$ is finite.

Two one-dimensional hypergroups $(H, *)$ and $(K, \circ)$ are equivalent if there is a continuous monotone $h \in C(H)$ such that $h(H)=K$ and for every $f \in$ $C(K)$

$$
\int_{K} f(z) d\left(\delta_{x} \circ \delta_{y}\right)(z)=\int_{H} f(h(r)) d\left(\delta_{s} * \delta_{t}\right)(r)
$$

where $s=h^{-1}(x)$ and $t=h^{-1}(y)$. In this case it will be said that $h$ is a domain transformation from $H$ to $K$ and that $K$ is obtained from $H$ by a change of variables. The two measure algebras are thus isometrically isomorphic. If

\footnotetext{
${ }^{1}$ W. C. Connett and A. L. Schwartz, A Hardy-Littlewood maximal inequality for Jacobi type hypergroups, Proc. Amer. Math. Soc. 107 (1989), 137-143.
} 
$(H, *)$ is differentiable and $h$ is infinitely differentiable and $h^{\prime} \neq 0$ then $(K, \circ)$ is differentiable also. Thus it will be no loss in the following to assume that $e=0$ unless required otherwise by context.

The following classification theorem is the main result of [17]:

Let $H$ be an interval or a circle and suppose that $(H, *)$ is a regular hypergroup. Then * must be a commutative operation and $(H, *)$ is equivalent to one of the following.

(i) $(G, *)$ where $G$ is either the circle group or the group of real numbers, * is the classical group convolution on $M(G)$, and $s^{\vee}$ is the group inverse.

(ii) $(K, *)$ where $K=[0, P]$ or $K=[0, \infty), e=0$, and $s^{\vee}=s$ for every $s \in K$.

$(H, *)$ is said to be of type I (resp. type II) if it is equivalent to a hypergroup which satisfies the hypotheses and conclusion (i) (resp. (ii)) of the theorem above. A type II hypergroup $(K, *)$ with $K=[0, P]$ is symmetric if $\delta_{P-t} *$ $\delta_{P-s}=\delta_{t} * \delta_{s}$ for every pair $s, t$ in $H$.

Since $*$ is a commutative operation it follows that $(H, *)$ has a positive measure $m$ such that $\operatorname{supp}(m)=H$ and $m * \delta_{t}=m$ for every $t \in H$ (see [18]); $m$ is called a Haar measure for $(H, *)$, and $m$ is unique up to a multiplicative constant. If $H$ is compact, it will usually be assumed that $m$ is normalized so that $m(H)=1$. If $f \in L^{p}(d m)$, the norm of $f$ is written $\|f\|_{p}$. The operators $T^{s}$ defined by

$$
T^{s} f(t)=\int_{H} f d\left(\delta_{s} * \delta_{t}\right)
$$

are called generalized translations because they satisfy the definition formulated in [13].

A function $\varphi$ in $C(H)$ which satisfies $\varphi\left(s^{\vee}\right)=\overline{\varphi(s)}$, and $T^{s}(\varphi)(t)=$ $\varphi(s) \varphi(t)$ for every $s, t \in H$ is called a Hermitian character of $(H, *)$. For $f \in L^{1}(d m)$, define $f^{\wedge}(\varphi)=\int_{H} f \varphi d m$, and let $H^{\curlywedge}$ denote the space of Hermitian characters. $H^{\wedge}$ is topologized so that each $f^{\wedge}$ is continuous on $\mathrm{H}^{\wedge}$, and there exists a measure $\mathrm{m}^{\wedge}$ on $\mathrm{H}^{\wedge}$, the so-called Plancherel measure, which satisfies

$$
\int_{H^{\wedge}}\left|f^{\wedge}\right|^{2} d m^{\wedge}=\int_{H}|f|^{2} d m \quad\left(f \in L^{2}(d m)\right) .
$$

In particular, if $H$ is compact then $H^{\wedge}$ constitutes a complete orthogonal system for $L^{2}(d m)$. (See [8, pp. 339-340] and the references cited there.)

It is now possible to define a multiplicative operation so that $L^{1}(\mathrm{dm})$ becomes a Banach algebra by setting

$$
f * g(s)=\int_{H} T^{s} f\left(t^{\vee}\right) g(t) d m(t) .
$$

This has the usual properties of such operations, in particular $\|f * g\|_{1} \leq$ $\|f\|_{1}\|g\|_{1}$ (see [12] for details and additional basic properties of convolution and hypergroups). 
If $k_{\mu}<\infty$, then there is a nontrivial function $A_{\mu}$ on the interior of $H$ such that for $t$ interior to $H$

$$
M_{\mu}(s, t)=M_{H, \mu}(s, t)=A_{\mu}(t) s^{k_{\mu}}+o\left(s^{k_{\mu}}\right) .
$$

It follows from commutativity and Lemma 1 of [17] that corresponding to the two classes of regular one-dimensional hypergroups are the conditions $1=k_{1}<$ $k_{2}$ for the type I hypergroups and $k_{2} \leq k_{1}$ for the type II hypergroups.

1.4. Continuation of Example $\mathbf{A}$. The characters of $(H, * ; \alpha, \beta)$ are $\left\{R_{n}\right\}$ (cf. equations (1.1) and (1.2)), $e=1$, and Haar measure is a constant multiple of $v$.

It will be useful to perform the domain transformation $s=\cos \theta$ so that $(H, * ; \alpha, \beta)$ becomes equivalent to a hypergroup $(K, \circ ; \alpha, \beta)$ with $K=$ $[0, \pi]$ and $e=0$. The properties of $(K, \circ ; \alpha, \beta)$ will be developed in some detail in order to motivate the definition of Jacobi type hypergroups and for comparison with the general theory to follow. The normalized Haar measure for $(K, \circ ; \alpha, \beta)$ is

$$
d m(\theta)=\rho^{2}(\theta) d \theta
$$

where

$$
\rho(\theta)=B(\sin \theta / 2)^{\alpha+1 / 2}(\cos \theta / 2)^{\beta+1 / 2}
$$

and

$$
B^{2}=\frac{\Gamma(\alpha+\beta+2)}{\Gamma(\alpha+1) \Gamma(\beta+1)}
$$

(see [19, equation (4.3.3)] with $n=0)$. The characters of $(K, \circ ; \alpha, \beta)$ are $\varphi_{n}(\theta)=R_{n}(\cos \theta)$, and the Plancherel measure, obtained easily from [19, equation (4.3.3)], is

$$
\widehat{m}(n)=h_{n}=\left\|\varphi_{n}\right\|_{2}^{-2}=\frac{(\alpha+1)_{n}(\alpha+\beta+1)_{n}}{(\beta+1)_{n} n !} \cdot \frac{2 n+\alpha+\beta+1}{\alpha+\beta+1}
$$

where $(c)_{0}=1$ and $(c)_{n}=c(c+1) \cdots(c+n-1)$.

The moments for $(K, \circ ; \alpha, \beta)$ are computed by first obtaining the moments for $(H, * ; \alpha, \beta)$ which is possible, though tedious, because the characters of $(H, * ; \alpha, \beta)$ are polynomials. The computation is only outlined here.

First, an explicit formula for $P_{n}^{(\alpha, \beta)}$ [19, equation (4.3.2)] is used to obtain constants $a, b, c$, and $d$ such that

$$
r-1=a\left(1-R_{1}(r)\right) \text { and } r^{2}=b R_{2}(r)+c R_{1}(r)+d ;
$$

then, by equation $(1.1)$

$$
\begin{aligned}
M_{H, 1}(s, t) & =\int_{H}(r-t) d \sigma_{s t}(r) \\
& =a \int_{H}\left[R_{1}(t)-R_{1}(r)\right] d \sigma_{s t}(r) \\
& =a\left[R_{1}(t)-R_{1}(s) R_{1}(t)\right]
\end{aligned}
$$


and

$$
\begin{aligned}
M_{H, 2}(s, t) & =\int_{H}(r-t)^{2} d \sigma_{s t}(r) \\
& =\int_{H}\left\{b R_{2}(r)+c R_{1}(r)+d-2 t a\left[1-R_{1}(r)\right]-2 t+t^{2}\right\} d \sigma_{s t}(r) \\
& =b R_{2}(s) R_{2}(t)+c R_{1}(s) R_{1}(t)+d-2 t a\left[1-R_{1}(s) R_{1}(t)\right]-2 t+t^{2} .
\end{aligned}
$$

Finally, substituting expressions for $R_{1}$ and $R_{2}$

$$
\begin{aligned}
& M_{H, 1}(s, t)=R_{1}(t)(s-1), \\
& M_{H, 2}(s, t)=\frac{1}{\alpha+1}\left(1-t^{2}\right)(1-s)+R_{2}(t)(1-s)^{2},
\end{aligned}
$$

and since $e=1$, it follows that $k_{1}=k_{2}=1$. The moments $M_{K, 1}$ and $M_{K, 2}$ for $(K, \circ ; \alpha, \beta)$ are obtained from (1.10) and (1.11) as follows; let $h(r)=\cos ^{-1}(r)$ so if $\psi=h(r)$ and $\varphi=h(t)$ then (cf. equation (1.6))

$$
\begin{aligned}
M_{K, 1}(\theta, \varphi) & =\int_{K}(\psi-\varphi) d\left(\delta_{\theta} \circ \delta_{\varphi}\right)(\psi) \\
& =\int_{H}[h(r)-h(t)] d\left(\delta_{s} * \delta_{t}\right)(r) \\
& =\int_{H}\left[h^{\prime}(t)(r-t)+\frac{1}{2} h^{\prime \prime}(t)(r-t)^{2}+O(r-t)^{3}\right] d\left(\delta_{s} * \delta_{t}\right)(r) \\
& =h^{\prime}(t) M_{H, 1}(s, t)+\frac{1}{2} h^{\prime \prime}(t) M_{H, 2}(s, t)+o(1-s)
\end{aligned}
$$

and similarly

$$
M_{K, 2}(\theta, \varphi)=\left[h^{\prime}(t)\right]^{2} M_{H, 2}(s, t)+o(1-s) .
$$

Finally substitute $s=\cos \theta, \quad t=\cos \varphi, h^{\prime}(t)=-1 / \sin \varphi$, and $h^{\prime \prime}(t)=$ $-\cos \varphi / \sin ^{3} \varphi$ to obtain

$$
\begin{aligned}
& M_{H, 1}(\theta, \varphi)=\frac{1}{\sin \varphi}\left[2 R_{1}(\cos \varphi)-\frac{\cos \varphi}{\alpha+1}\right] \sin ^{2} \frac{\theta}{2}+o\left(\theta^{2}\right), \\
& M_{H, 2}(\theta, \varphi)=\frac{2}{\alpha+1} \sin ^{2} \frac{\theta}{2}+o\left(\theta^{2}\right)
\end{aligned}
$$

so

$$
k_{1}=k_{2}=2 \quad A_{1}(\varphi)=\frac{1}{4 \sin \varphi}\left[2 R_{1}(\cos \varphi)-\frac{\cos \varphi}{\alpha+1}\right]
$$

and

$$
A_{2}(\varphi)=a_{2}=\frac{1}{2(\alpha+1)} .
$$

Additional properties of $(K, \circ ; \alpha, \beta)$ will be discussed in $\S 5$.

\section{JACOBI-TYPE HYPERGROUPS}

For the balance of the paper we require all hypergroups to be differentiable in the slightly stronger sense that if $f \in C(H)$ is $p$ times continuously differentiable, then $u(s, t)=T^{s} f(t)$ has bounded $p$ th order derivatives on the interior 
of $H \times H$. This added requirement may be replaced by the assumption that the span of $H^{-}$is uniformly dense in $C(H)$, or that $(H, *)$ is a symmetric hypergroup, or by more complicated (but less restrictive hypotheses). See Remark 4.9.

The Jacobi example motivates the following definition:

If $(\alpha, \beta)$ is a pair of real numbers, a hypergroup $(H, *)$ is of Jacobi type $(\alpha, \beta)$ if it is equivalent to a type II hypergroup in which:

J1. $H=[0, \pi]$.

J2. $k_{1}=k_{2}=2$.

J3. $\sin t A_{1}(t)$ can be extended to a differentiable function on $H$.

J4. $A_{2}(t)=a_{2}>0$.

J5. $\lim _{t \rightarrow 0+} a_{2}^{-1} \sin t A_{1}(t)=\alpha+\frac{1}{2}$ and $\lim _{t \rightarrow \pi-} a_{2}^{-1} \sin t A_{1}(t)=-\left(\beta+\frac{1}{2}\right)$.

The hypergroups $(K, \circ ; \alpha, \beta)$ described in the example are of Jacobi type $(\alpha, \beta)$ provided $(\alpha, \beta)$ satisfies Gasper's conditions of $\alpha \geq \beta$ and either $\beta \geq-\frac{1}{2}$ or $\alpha+\beta \geq 0$.

The definition is not so restrictive as it may seem at first glance. The following theorems, based on changes of variables, indicate the generality of the class.

2.1. Theorem. Let $(H, *)$ be a type II hypergroup for which

(i) $H=[0, P]$.

(ii) $k_{1}=k_{2}=2$.

(iii) $\sin \left(P^{-1} \pi t\right) A_{1}(t)$ and $A_{2}(t)$ can be extended to differentiable functions on $H$ with $A_{2}$ positive on $H$.

(iv)

$$
\lim _{t \rightarrow 0+} \frac{t A_{1}(t)}{A_{2}(t)}=\alpha+\frac{1}{2} \quad \text { and } \quad \lim _{t \rightarrow P-} \frac{(t-P) A_{1}(t)}{A_{2}(t)}=\beta+\frac{1}{2} .
$$

Then $(H, *)$ is a Jacobi type $(\alpha, \beta)$ hypergroup.

Proof. Let $(H, *)$ satisfy the hypotheses of the lemma and define a domain transformation by letting $a(t)=\int_{0}^{t}\left[A_{2}(r)\right]^{-1 / 2} d r$ and $y=h(t)=\pi a(t) / a(P)$, then $(H, *)$ is equivalent to $(K, \circ)$ where $K=[0, \pi]$ and $\circ$ is defined by equation (1.6). Moreover, proceeding as in the example

$$
\begin{gathered}
M_{K, 1}(x, y)=h^{\prime}(t) M_{H, 1}(s, t)+\frac{1}{2} h^{\prime \prime}(t) M_{H, 2}(s, t)+o\left(s^{2}\right), \\
M_{K, 2}(x, y)=\left[h^{\prime}(t)\right]^{2} M_{H, 2}(s, t)+o\left(s^{2}\right),
\end{gathered}
$$

so

$$
M_{K, 1}(x, y)=B_{1}(y) x^{2}+o\left(x^{2}\right)
$$

and

$$
M_{K, 2}(x, y)=A_{2}(0) x^{2}+o\left(x^{2}\right)
$$

where, if $t=h^{-1}(y)$,

$$
B_{1}(y)=\frac{a(P)}{\pi} \frac{A_{2}(0)}{\left[A_{2}(t)\right]^{1 / 2}}\left[A_{1}(t)-\frac{1}{4} A_{2}^{\prime}(t)\right] .
$$


Finally, with $a_{2}=A_{2}(0)$,

$$
\begin{gathered}
\lim _{y \rightarrow 0+} a_{2}^{-1} \sin y B_{1}(y)=\alpha+\frac{1}{2}, \\
\lim _{y \rightarrow \pi_{-}} a_{2}^{-1} \sin y B_{1}(y)=-\left(\beta+\frac{1}{2}\right) .
\end{gathered}
$$

The following theorem is exhaustive with regard to $\left(k_{1}, k_{2}\right)$ because $k_{1} \geq k_{2}$ for every type II hypergroup.

2.2. Theorem. Let $(H, *)$ be a type II hypergroup for which

(i) $H=[0, P]$.

(ii) $A_{2}(t)$ can be extended to a positive differentiable function on $H$, and if $k_{1}=k_{2}$ then $\sin \left(P^{-1} \pi t\right) A_{1}(t)$ can be extended to a differentiable function on $H$.

(iii) If $k_{1}=k_{2}$ then

$$
\lim _{t \rightarrow 0+} \frac{2 t A_{1}(t)}{k_{2} A_{2}(t)}=\alpha+k_{2}^{-1} \text { and } \lim _{t \rightarrow P-} \frac{2(t-P) A_{1}(t)}{k_{2} A_{2}(t)}=\beta+k_{2}^{-1},
$$

and if $k_{1}>k_{2}$ then $\alpha=\beta=-k_{2}^{-1}$.

Then $(H, *)$ is a Jacobi type $(\alpha, \beta)$ hypergroup.

Proof. Proceed as in Theorem 2.1 with $y=h(t)=t^{k_{2} / 2}$, then

$$
M_{K, \mu}=B_{\mu}(y) x^{2}+o\left(x^{2}\right) \quad(\mu=1,2)
$$

with

$$
\begin{aligned}
& B_{1}(y)=\frac{1}{2} h^{\prime \prime}(t) A_{2}(t)+\varepsilon h^{\prime}(t) A_{1}(t), \\
& B_{2}(y)=\left[h^{\prime}(t)\right]^{2} A_{2}(t),
\end{aligned}
$$

where $\varepsilon=1$ if $k_{1}=k_{2}$ and $\varepsilon=0$ otherwise. It is easy to show that $B_{1}$ and $B_{2}$ satisfy the hypotheses of $A_{1}$ and $A_{2}$ in Theorem 2.1 so the result follows.

\section{Differential equations}

It will be assumed for the rest of the paper that $(H, *)$ is a Jacobi type $(\alpha, \beta)$ hypergroup. Let $B(t)=A_{1}(t) / a_{2}$ then from $\mathrm{J} 5$

$$
\lim _{t \rightarrow 0+} \sin t B(t)=\alpha+\frac{1}{2} \text { and } \lim _{t \rightarrow \pi-} \sin t B(t)=-\left(\beta+\frac{1}{2}\right)
$$

If $f \in C^{2}(H)$, then $u(s, t)=T^{s} f(t)$ solves the following Cauchy problem:

$$
\left.\begin{array}{rr}
u_{s s}+2 B(s) u_{s}=u_{t t}+2 B(t) u_{t} & (0<s, t<\pi) \\
u(0, t)=f(t), \quad u_{s}(0, t)=0 & (0<t<\pi)
\end{array}\right\}
$$

(see [13, II.6] and [17]).

Two more partial differential equations related to equation (3.2) will be required. Define

$$
\rho(t)=c \exp \left[\int_{\pi / 2}^{t} B(r) d r\right]
$$


where $c$ is chosen so that $\int_{0}^{\pi} \rho^{2}(t) d t=1$. Let $W(s, t)=\rho^{2}(s) \rho^{2}(t)$, then if $u$ is a solution of equation (3.2)

$$
\frac{\partial}{\partial s}\left(W \frac{\partial u}{\partial s}\right)=\frac{\partial}{\partial t}\left(W \frac{\partial u}{\partial t}\right)
$$

Finally, if $q=\rho^{\prime \prime} / \rho$ and if $v(s, t)=\rho(s) \rho(t) u(s, t)$, then

$$
\frac{\partial^{2} v}{\partial s^{2}}-q(s) v=\frac{\partial^{2} v}{\partial t^{2}}-q(t) v
$$

An important consequence of equation (3.2) is that the characters of $(H, *)$ are eigenfunctions of a second order linear differential operator. This fact, expressed in Theorem 3.5 below, plays a key role in understanding the properties of characters. First, however, some information is required about the behavior of $\rho$ and $q$ near 0 and $\pi$.

3.1 Lemma. (i) There are positive constants $a_{\alpha}$ and $a_{\beta}$ such that

$$
\lim _{t \rightarrow 0+} t^{-\alpha-1 / 2} \rho(t)=a_{\alpha} \text { and } \lim _{t \rightarrow \pi-}(\pi-t)^{-\beta-1 / 2} \rho(t)=a_{\beta} .
$$

(ii)

$$
q(t)=\frac{\alpha^{2}-1 / 4}{t^{2}}+\frac{\beta^{2}-1 / 4}{(\pi-t)^{2}}+Q(t),
$$

where $\sin t Q(t)$ is a bounded continuous function on $(0, \pi)$. In the limiting case $\alpha=-\frac{1}{2}$ (resp. $\left.\beta=-\frac{1}{2}\right), Q$ is actually continuous at 0 (resp. $\pi$ ).

Proof. (i) follows from equations (3.1) and (3.3). To prove (ii), use equation (3.3) to obtain $q=B^{2}+B^{\prime}$, and then employ equation (3.1).

3.2 Lemma. If $\varphi$ belongs to $H^{-}$then $\varphi$ is a real-valued twice continuously differentiable function and for some $\mu \geq 0, y=\varphi$ is a solution of the initial value problem

$$
\left(\rho^{2} y^{\prime}\right)^{\prime}+\mu \rho^{2} y=0, \quad y(0)=1, \quad y^{\prime}(0)=0 .
$$

Proof. If $\varphi \in H^{\wedge}$, then, since $s^{\vee}=s, \varphi(s)=\varphi\left(s^{\vee}\right)=\overline{\varphi(s)}$ so $\varphi$ must be a real-valued function. Also if $f \in C^{2}(H)$ then, as in [13, pp. 65-66],

$$
\varphi(s) \int_{0}^{\pi} \varphi(t) f(t) d m(t)=\int_{0}^{\pi} T^{s} \varphi(t) f(t) d m(t)=\int_{0}^{\pi} \varphi(t) T^{s} f(t) d m(t),
$$

the last expression is twice continuously differentiable with respect to $S$, so $\varphi \in C^{2}(H)$ and hence $u(s, t)=T^{s} \varphi(t)=\varphi(s) \varphi(t)$ solves (3.4). Separation of variables shows that (3.6) holds for some real number $\mu$ and $y=\varphi$. The first initial condition, $\varphi(0)=1$, must hold since $\varphi$ is a character and $\delta_{0}$ is the identity element of $(H, *)$; the second condition, $\varphi^{\prime}(0)=0$, follows from the boundary condition $u_{s}(0, t)=0$ specified in (3.2).

To show that $\mu \geq 0$, assume by way of contradiction that $\mu<0$. Since $\varphi(0)=1, \varphi$ is positive on $N=(0, \varepsilon)$ for some $\varepsilon>0$, so $\left(\rho^{2} \varphi^{\prime}\right)^{\prime}=-\mu \rho^{2} \varphi>0$ 
on $N$. This implies that $\rho^{2} \varphi^{\prime}$ is strictly increasing on $N$, hence $\varphi^{\prime}$ is positive on $N$ because $\varphi^{\prime}(0)=0$. Since $\varphi(0)=1$, it follows that $\varphi$ exceeds 1 on $N$ which is impossible since $\varphi$ is a character, and necessarily bounded by 1 . This contradiction establishes that $\mu \geq 0$.

It will be useful to have some properties of (3.6). The following lemma is a slight generalization of [1, Chapter 8 , Theorem 2.1] which is stated for regular differential equations, but the proof given is applicable to the present situation.

\subsection{Lemma. Consider the Sturm-Liouville problem}

$$
\begin{gathered}
\left(\rho^{2} y^{\prime}\right)^{\prime}+\nu \rho^{2} y=0, \\
y^{\prime}(0)=0, \quad \lim _{t \rightarrow \pi-}\left[C_{1} y(t)+C_{2} \rho^{2}(t) y^{\prime}(t)\right]=0
\end{gathered}
$$

where at least one of $C_{1}$ and $C_{2}$ is not zero. Then the eigenvalues may be arranged in an unbounded increasing sequence $\nu_{0}<\nu_{1}<\nu_{2}<\cdots$ and if $y_{k}$ is a nontrivial eigenfunction corresponding to $\nu_{k}$, then $y_{k}$ has exactly $k$ zeros in $(0, \pi)$.

The following asymptotic estimate of eigenvalues will also be required.

3.4 Lemma. Let $\nu_{k}$ be as in Lemma 3.3, then $\lim _{k \rightarrow \infty} k^{-2} \nu_{k}=1$.

Proof. It will suffice to show there are constants $c$ and $C$ such that

$$
\begin{aligned}
& k^{-2} \nu_{k} \leq 1+C k^{-1}, \\
& k^{2} \nu_{k}^{-1} \leq 1+c \nu_{k}^{-1 / 2},
\end{aligned}
$$

and the result will follow because the sequence $\nu_{k}$ is unbounded (Lemma 3.3). To do this, we will show that if $k \in E=\left\{p: y_{p}\right.$ has no more than $p / 2$ zeros in $(0, \pi / 2)\}$, the first inequality holds, and if $k \notin E$, the second holds. Those arguments with $t$ and $\alpha$ replaced by $\pi-t$ and $\beta$ respectively lead to the conclusion that the inequalities hold for every $k$.

Let $y_{k}$ be a nontrivial eigenfunction corresponding to $\nu_{k}$. Let $j_{0}, j_{1}$, $j_{2}, \ldots$ denote the positive zeros of $J_{\alpha}(t)$ arranged in ascending order, let $m_{p}=$ $2 \pi^{-1} j_{p}$, and let $I_{p}=\left(m_{0} / m_{p}, \pi / 2\right)$. Then $J_{\alpha}\left(m_{p} t\right)$ has exactly $p$ zeros in $I_{p}$, and from $[21,15.53]$ (or more simply (4.8) below)

$$
m_{p}=2 p+O(1) \text {. }
$$

Let $k$ belong to $E$, and let $n$ be the greatest integer not exceeding $k / 2$. Then there cannot be a zero of $y_{k}$ between every pair of zeros of $J_{\alpha}\left(m_{n+2} t\right)$ in $I_{n+2}$.

Now recall that $y(t)=t^{1 / 2} J_{\alpha}\left(m_{n+2} t\right)$ satisfies

$$
y^{\prime \prime}+\left(m_{n+2}^{2}-\frac{\alpha^{2}-1 / 4}{t^{2}}\right) y=0,
$$

so the Sturm Comparison Theorem [1, p. 208] implies that there exists $\xi \in I_{n+2}$ such that

$$
m_{n+2}^{2}-\left(\alpha^{2}-\frac{1}{4}\right) \xi^{-2} \geq \nu_{k}-q(\xi)
$$


or more simply, $m_{n+2}^{2} \geq \nu_{k}-Q_{\alpha}(\xi)$ where $Q_{\alpha}(\xi)=q(\xi)-\left(\alpha^{2}-\frac{1}{4}\right) \xi^{-2}$. Further, $\xi Q_{\alpha}(\xi)$ is bounded in $I_{n+2}$ (cf. Lemma 3.1), so there is a constant $C_{1}$ such that $\left|Q_{\alpha}(\xi)\right| \leq C_{1} m_{n+2} / m_{0}$. Equation (3.9) implies there is a constant $C_{2}$ independent of $n$ such that $m_{n+2} \leq k+C_{2}$. The last three inequalities yield $\left(k+C_{2}\right)^{2} \geq \nu_{k}-C_{1}\left(k+C_{2}\right) / m_{0}$. Division by $k^{2}$ shows there is a constant $C$ such that (3.7) holds.

Let $\mu \geq 0$ and assume that $y$ is the solution of the initial value problem (3.6). Let $z(t)=z(\lambda ; t)=y(t / \lambda)$ where $\lambda^{2}=\mu$. Then $z$ satisfies the initial value problem

$$
\begin{gathered}
z^{\prime \prime}+2 t^{-1} b(t / \lambda) z^{\prime}+z=0 \\
z(0)=1 \text { and } z^{\prime}(0)=0
\end{gathered}
$$

where $b(t)=t \rho^{\prime}(t) / \rho(t)$ has a limit as $t \rightarrow 0+($ see equations (3.1) and (3.3)). It follows that there is a constant $C_{3}>0$ such that

$$
z(\lambda ; t)>0 \quad\left(0<t<C_{3}\right) .
$$

Now for sufficiently large $k, \nu_{k} \geq 0$ so there exists $\lambda_{k} \geq 0$ such that $\nu_{k}=$ $\lambda_{k}^{2}$. Thus (3.10) implies $y_{k}(t)=z\left(\lambda_{k} ; t\right) \neq 0$ if $t \in I^{k}=\left(C_{3} / \lambda_{k}, \pi / 2\right)$. If $k \notin E$ and $n$ is as above $y_{k}$ has more than $n$ zeros in $I^{k}$ because of Lemma 3.3. Thus the zeros of $J_{\alpha}\left(m_{n-1} t\right)$ cannot interlace those of $y_{k}$ in $I^{k}$. Proceeding as above, there exists $\xi \in I^{k}$ such that $m_{n-1}^{2} \leq \nu_{k}-Q_{\alpha}(\xi)$ and there is a constant $C_{4}$ such that $m_{n-1} \geq k-C_{4}$ so $\left(k-C_{4}\right)^{2} \leq \nu_{k}+C_{1} \lambda_{k} / C_{3}$ hence (3.8) holds for some constant $c$.

3.5 Theorem. There is an enumeration $\varphi_{0}=1, \varphi_{1}, \varphi_{2}, \ldots$ of $H^{\wedge}$ and an unbounded increasing sequence of real numbers $0=\lambda_{0}<\lambda_{1}<\lambda_{2}<\cdots$ such that $y=\varphi_{k}$ and $\mu=\lambda_{k}^{2}$ solves the initial value problem (3.6). The Plancherel measure for $H^{-}$is given by $h_{k}=\left\|\varphi_{k}\right\|_{2}^{-2}$ in the sense that

$$
\int_{0}^{\pi}|f|^{2} d m=\sum_{k=0}^{\infty}\left|f^{\wedge}\left(\varphi_{k}\right)\right|^{2} h_{k} .
$$

Proof. $H^{\curlywedge}$ is a countable orthogonal basis for $L^{2}(\mathrm{dm})$ because $H$ is compact [8, Theorem 3.5]. $H^{\wedge}$ is topologized so that $f^{\wedge}$ is continuous for every $f \in$ $L^{1}(d m)$. In particular, if $\varphi \in H^{\wedge}$ and $f(t)=\varphi(t)$, it follows that $\{\varphi\}$ is an open subset of $H^{\wedge}$, so that $H^{\wedge}$ has the discrete topology.

Thus $M=\left\{\mu:(3.6)\right.$ holds for some $\left.y \in H^{\wedge}\right\}$ is a countable set with no accumulation points. Lemma 3.2 implies $M \subseteq[0, \infty)$, so by Lemma $3.3 M$ can be enumerated as an unbounded increasing sequence $0 \leq \mu_{0}<\mu_{1}<\mu_{2}$ $<\cdots$.

Let $\varphi(t)=1 \quad(t \in H)$. Since $\delta_{s} * \delta_{t}$ is a probability measure,

$$
\int_{H} \varphi(u) d\left(\delta_{s} * \delta_{t}\right)(u)=1=\varphi(s) \varphi(t)
$$


so $\varphi$ is a character and the corresponding eigenvalue from (3.6) is 0 . Thus $\varphi_{0}=1$ and $\mu_{0}=0$.

Frequently, as in Examples B and C, a hypergroup is obtained because the eigenfunctions of a Sturm-Liouville problem satisfy a product formula analogous to (1.1) with nonnegative measures $\sigma_{s t}$. The characters of the resulting hypergroup are normalized eigenfunctions.

The following theorem shows that the hypergroup may be classified by direct reference to the differential operator without resorting to the calculation of moments. Its proof will be deferred to the end of the next section.

3.6 Theorem. Suppose $(H, *)$ is a type II hypergroup with $H=[0, \pi]$ and suppose the characters are also eigenfunctions of a Sturm-Liouville problem on $H$ :

$$
\begin{gathered}
\left(w y^{\prime}\right)^{\prime}+\lambda^{2} w y=0, \\
y^{\prime}(0)=0 \quad \text { and } \lim _{t \rightarrow \pi-}\left[C_{1} y(t)+C_{2} w(t) y^{\prime}(t)\right]=0
\end{gathered}
$$

where $w$ is a positive continuously differentiable function on $(0, \pi)$ such that $\lim _{t \rightarrow 0+} t^{-2 \alpha-1} w(t)$ and $\lim _{t \rightarrow 0+}(\pi-t)^{-2 \beta-1} w(t)$ exist and are positive, then $(H, *)$ is a hypergroup of Jacobi type $(\alpha, \beta), w(t)=c \rho^{2}(t)$ where $\rho$ is defined by (3.3) and $c$ is a constant, and the characters of $(H, *)$ form a complete set of eigenfunctions for the problem (3.11)-(3.12). The same result holds if (3.11) is replaced by $y^{\prime \prime}+2 b y^{\prime}+\lambda^{2} y=0$ and the assumption that (3.1) holds with $b$ in place of $B$.

\section{Properties of the characters and the Plancherel measure}

In this section, the fact that the characters satisfy a differential equation related to Bessel's equation is exploited to obtain asymptotic estimates of the characters and the Plancherel measure. For the sake of simplicity in exposition it will be assumed in this section that $\alpha$ and $\beta$ are nonnegative. With some small modifications, all of the results are true if $-\frac{1}{2} \leq \alpha<0$ or $-\frac{1}{2} \leq \beta<0$ with nearly the same arguments. The necessary changes are given in $\S 4.8$.

Let $J_{\nu}$ be the Bessel function of the first kind of order $\nu$ and let $\mathscr{J}_{\nu}(z)=$ $2^{\nu} \Gamma(\nu+1) z^{-\nu} J_{\nu}(z)$ so that $\mathscr{J}_{\nu}$ is an entire function with $\mathscr{J}_{\nu}(0)=1$.

In the arguments of this section $C$ will denote a positive constant which need not be the same at every occurrence. The following Hilb-type estimate is inspired by [19, Theorem 8.21.12].

4.1 Theorem. Let $a_{\alpha}$ and $a_{\beta}$ be as in Lemma 3.1 and let $\varepsilon>0$. Then there is a constant $C$ depending only on $\alpha, \beta$, and $\varepsilon$ such that:

(i)

$$
\varphi_{k}(s)=\left[a_{\alpha} s^{\alpha+1 / 2} / \rho(s)\right] \mathscr{J}_{\alpha}\left(\lambda_{k} s\right)+I_{k}(s)
$$


with

$$
\varphi_{k}(s)=\varphi_{k}(\pi)\left\{\left[a_{\beta}(\pi-s)^{\beta+1 / 2} / \rho(s)\right] \mathscr{J}_{\beta}\left(\lambda_{k}(\pi-s)\right\}+M_{k}(s)\right.
$$

with

$$
\begin{array}{ll}
\left|M_{k}(s)\right| \leq C \lambda_{k}^{-1} & \left(0 \leq \pi-s \leq \lambda_{k}^{-1}\right), \\
\left|M_{k}(s)\right| \leq C \lambda_{k}^{-1}\left[\lambda_{k}(\pi-s)\right]^{-\beta-1 / 2} \ln \lambda_{k} & \left(\varepsilon \leq s \leq \pi-\lambda_{k}^{-1}\right) .
\end{array}
$$

Proof. It will be assumed in this proof that $\alpha$ is not an integer. When $\alpha$ is an integer, $J_{-\alpha}$ can be replaced at each appearance by $Y_{\alpha}$, the Bessel function of the second kind of order $\alpha$, and the analysis is quite similar.

From Theorem 3.5 and Lemma 3.1(ii) $y=a_{\alpha}^{-1} \rho \varphi_{k}$ solves

$$
y^{\prime \prime}+\left(\lambda_{k}^{2}-\frac{\alpha^{2}-1 / 4}{s^{2}}\right) y=Q_{\alpha} y
$$

where $s Q_{\alpha}(s)$ is bounded on $[0, \pi-\varepsilon]$ for every $\varepsilon>0$.

By the method of variation of parameters [10 or $19, \S 8.63]$

$$
\begin{aligned}
y(s)= & A_{k} s^{1 / 2} J_{\alpha}\left(\lambda_{k} s\right)+B_{k} s^{1 / 2} J_{-\alpha}\left(\lambda_{k} s\right) \\
& +\frac{\pi}{2 \sin \alpha \pi} \int_{0}^{s}(s t)^{1 / 2} K_{k}(s, t) Q_{\alpha}(t) y(t) d t
\end{aligned}
$$

where

$$
K_{k}(s, t)=J_{\alpha}\left(\lambda_{k} s\right) J_{-\alpha}\left(\lambda_{k} t\right)-J_{\alpha}\left(\lambda_{k} t\right) J_{-\alpha}\left(\lambda_{k} s\right) .
$$

Since $\alpha$ is nonnegative, the contribution of the second term in (4.5) to $\varphi_{k}$ would be unbounded for a nonzero $B_{k}$ (it will be shown below that the contribution of the third term to $\varphi_{k}$ is bounded), thus $B_{k}=0$ and (4.1) holds with

$$
I_{k}(s)=\int_{0}^{s} H_{k}(s, t) \varphi_{k}(t) d t
$$

where

$$
H_{k}(s, t)=\frac{\pi}{2 \sin \alpha \pi}\left(\frac{s}{t}\right)^{1 / 2} \frac{\rho(t)}{\rho(s)} K_{k}(s, t) t Q_{\alpha}(t) .
$$

The standard estimates $J_{\alpha}(x)=O\left(x^{\alpha}\right) \quad(0<x<1)$ and $J_{\alpha}(x)=O\left(x^{-1 / 2}\right)$ $(x>1)$, the bound $t Q_{\alpha}(t)=O(1)$, and Lemma 3.1(i) can be used to obtain

$$
\left|H_{k}(s, t)\right| \leq \begin{cases}C & \left(0 \leq t \leq s \leq \lambda_{k}^{-1}\right) \\ C\left(\lambda_{k} s\right)^{-\alpha-1 / 2} & \left(0 \leq t \leq \lambda_{k}^{-1} \leq s\right) \\ C(t / s)^{\alpha+1 / 2}\left(\lambda_{k} t\right)^{-1} & \left(0 \leq \lambda_{k}^{-1} \leq t \leq s\right)\end{cases}
$$


Equation (4.2) is now obtained since $\left\|\varphi_{k}\right\|_{\infty}=1$.

To obtain (4.3) use (4.1) and (4.6) to write $I_{k}(s)=S_{1}(s)+S_{2}(s)+S_{3}(s)$ where

$$
\begin{aligned}
& S_{1}(s)=\int_{0}^{1 / \lambda_{k}} H_{k}(s, t) \varphi_{k}(t) d t \\
& S_{2}(s)=\int_{1 / \lambda_{k}}^{s} H_{k}(s, t)\left[a_{\alpha} t^{\alpha+1 / 2} / \rho(t)\right] \mathcal{J}_{\alpha}\left(\lambda_{k} t\right) d t \\
& S_{3}(s)=\int_{1 / \lambda_{k}}^{s} H_{k}(s, t) I_{k}(t) d t
\end{aligned}
$$

and define $F_{k}=\sup \left|t^{\alpha+1 / 2} I_{k}(t)\right|\left(\lambda_{k} t \geq 1\right)$. Now use $\left\|\varphi_{k}\right\|_{\infty}=1$ and (4.7) to obtain

$$
s^{\alpha+1 / 2}\left|S_{1}(s)\right| \leq C \lambda_{k}^{-\alpha-3 / 2} \text {. }
$$

To estimate $S_{2}(s)$ use $\left|\mathscr{J}_{\alpha}\left(\lambda_{k} t\right)\right| \leq K\left(\lambda_{k} t\right)^{-\alpha-1 / 2}$ and find

$$
s^{\alpha+1 / 2}\left|S_{2}(s)\right| \leq C \lambda_{k}^{-\alpha-3 / 2} \ln \lambda_{k},
$$

and finally

$$
s^{\alpha+1 / 2}\left|S_{3}(s)\right| \leq C F_{k} \lambda_{k}^{-1} \ln \lambda_{k}
$$

so if $\lambda_{k} s \geq 1$

$$
\left|s^{\alpha+1 / 2} I_{k}(s)\right|=\left|s^{\alpha+1 / 2} S_{1}(s)+s^{\alpha+1 / 2} S_{2}(s)+s^{\alpha+1 / 2} S_{3}(s)\right|
$$

therefore,

$$
F_{k} \leq C \ln \lambda_{k}\left[\lambda_{k}^{-\alpha-3 / 2}+\lambda_{k}^{-1} F_{k}\right]
$$

whence

$$
F_{k} \leq C \ln \lambda_{k} / \lambda_{k}^{\alpha+3 / 2}
$$

since $\lambda_{k} \rightarrow \infty$ as $k \rightarrow \infty$, and (4.3) is proved.

The proof of part (ii) is similar to the above but with $s$ and $\alpha$ replaced by $\pi-s$ and $\beta$.

4.2 Corollary. $\varphi_{k}^{\prime}(0)=\varphi_{k}^{\prime}(\pi)=0$.

Proof. This is a simple consequence of the fact that $B_{k}=0$ in (4.5) and in the corresponding equation with $s$ and $\alpha$ replaced by $\pi-s$ and $\beta$.

Let $E_{\alpha}=2^{\alpha} \Gamma(\alpha+1) a_{\alpha}, E_{\beta}=2^{\beta} \Gamma(\beta+1) a_{\beta}$, and $E=E_{\alpha} / E_{\beta}$.

4.3 Theorem. (i) $\lim _{k \rightarrow \infty} \lambda_{k}^{\alpha-\beta}\left|\varphi_{k}(\pi)\right|=E$.

(ii) $\alpha \geq \beta$.

(iii) $\lim _{k \rightarrow \infty} \lambda_{k}^{2 \alpha+1} \int_{0}^{\pi} \varphi_{k}^{2}(t) \rho^{2}(t) d t=E_{\alpha}^{2}$.

(iv) There is a constant $K \geq 0$ such that $\varphi_{k}(t) \geq \frac{1}{3}$ if $\lambda_{k} t \leq K$. 
Proof. Suppose $\left|\frac{\pi}{2}-s\right|<\frac{\pi}{4}$, then Theorem 4.1 yields

$$
\begin{aligned}
& a_{\alpha} \frac{s^{\alpha+1 / 2}}{\rho(s)} \mathscr{J}_{\alpha}\left(\lambda_{k} s\right)+o\left(\lambda_{k}^{-\alpha-1 / 2}\right) \\
& \quad=\varphi_{k}(\pi)\left[a_{\beta} \frac{(\pi-s)^{\beta+1 / 2}}{\rho(s)} \mathscr{J}_{\beta}\left(\lambda_{k}(\pi-s)\right)+o\left(\lambda_{k}^{-\beta-1 / 2}\right)\right]
\end{aligned}
$$

whence, using the definition of $\mathscr{J}_{\nu}$, if $\varphi_{k}(s) \neq 0$

$$
\lambda_{k}^{\alpha-\beta} \varphi_{k}(\pi)=\frac{E[\rho(s)]^{-1}\left(\lambda_{k} s\right)^{1 / 2} J_{\alpha}\left(\lambda_{k} s\right)+o(1)}{[\rho(s)]^{-1}\left[\lambda_{k}(\pi-s)\right]^{1 / 2} J_{\beta}\left(\lambda_{k}(\pi-s)\right)+o(1)} .
$$

The asymptotic estimate $[21,7.21(1)]$

$$
J_{\nu}(z)=\left(\frac{2}{\pi z}\right)^{1 / 2} \cos \left(z-\frac{\nu \pi}{2}-\frac{\pi}{4}+O\left(z^{-3 / 2}\right)\right)
$$

can be used to obtain

$$
E^{-1} \lambda_{k}^{\alpha-\beta} \varphi_{k}(\pi)=\frac{\cos \left(\lambda_{k} s_{k}-\alpha \pi / 2-\pi / 4\right)+\rho(s) o(1)}{\cos \left[\lambda_{k}\left(\pi-s_{k}\right)-\pi / \beta 2-\pi / 4\right]+\rho(s) o(1)} .
$$

Now let $\varepsilon>0$ and choose $s_{k}, k=1,2, \ldots$, so that

$$
\cos \left[\lambda_{k}\left(\pi-s_{k}\right)-\frac{\beta \pi}{2}-\frac{\pi}{4}\right]=1
$$

and it follows that $\left|E^{-1} \lambda_{k}^{\alpha-\beta} \varphi_{k}(\pi)\right| \leq 1+\varepsilon$ if $k$ is sufficiently large. Similarly if $s_{k}$ are chosen so that

$$
\cos \left[\lambda_{k} s_{k}-\frac{\alpha \pi}{2}-\frac{\pi}{4}\right]=1
$$

then $\left|E^{-1} \lambda_{k}^{\alpha-\beta} \varphi_{k}(\pi)\right| \geq 1-\varepsilon$ if $k$ is sufficiently large. Thus $\left|\lambda_{k}^{\alpha-\beta} \varphi_{k}(\pi)\right| \rightarrow E$ as $k \rightarrow \infty$.

To obtain (ii) observe that each $\varphi_{k}$ is a character hence $\left|\varphi_{k}(\pi)\right| \leq 1$ for all $k$; this will be violated unless $\alpha \geq \beta$.

For part (iii) let

$$
\begin{aligned}
& S_{\alpha}(k)=\lambda_{k}^{2 \alpha+1} \int_{0}^{\pi / 2} \varphi_{k}^{2}(s) \rho^{2}(s) d s, \\
& T_{1}(k)=\lambda_{k}^{2 \alpha+1} \int_{0}^{1 / \lambda_{k}} \varphi_{k}^{2}(s) \rho^{2}(s) d s, \\
& T_{2}(k)=\lambda_{k}^{2 \alpha+1} \int_{1 / \lambda_{k}}^{\pi / 2}\left\{\left[a_{\alpha} s^{\alpha+1 / 2} / \rho(s)\right] \mathscr{F}_{\alpha}\left(\lambda_{k} s\right)\right\}^{2} \rho^{2}(s) d s, \\
& T_{3}(k)=\lambda_{k}^{2 \alpha+1} \int_{1 / \lambda_{k}}^{\pi / 2} I_{k}^{2}(s) \rho^{2}(s) d s
\end{aligned}
$$

where $I_{k}$ has the same meaning as in Theorem 4.1. 
Now, since $\left\|\varphi_{k}\right\|_{\infty}=1$ and $\rho(s)=O\left(s^{\alpha+1 / 2}\right), T_{1}(k)=O\left(\lambda_{k}^{-1}\right)$ and $T_{3}(k)=$ $O\left(\left(\lambda_{k}^{-1} \ln \lambda_{k}\right)^{2}\right)$, so

$$
\lim _{k \rightarrow \infty} T_{1}(k)=\lim _{k \rightarrow \infty} T_{3}(k)=0
$$

Also

$$
\begin{aligned}
T_{2}(k) & =\int_{1 / \lambda_{k}}^{\pi / 2}\left[E_{\alpha} J_{\alpha}\left(\lambda_{k} s\right)\right]^{2} \lambda_{k} s d s \\
& =E_{\alpha}^{2}\left(\lambda_{k}\right)^{-1} \int_{1}^{\lambda_{k} \pi / 2} J_{\alpha}^{2}(u) u d u,
\end{aligned}
$$

thus (cf. $[21,5.11(11)]) \lim _{k \rightarrow \infty} T_{2}(k)=E_{\alpha}^{2} / 2>0$. Thus

$$
\lim _{k \rightarrow \infty} S_{\alpha}(k)=\lim _{k \rightarrow \infty} T_{2}(k)=E_{\alpha}^{2} / 2 \text {. }
$$

If

$$
S_{\beta}(k)=\lambda_{k}^{2 \beta+1} \int_{\pi / 2}^{\pi}\left[\varphi_{k}(s) / \varphi_{k}(\pi)\right]^{2} \rho^{2}(s) d s
$$

then the same argument as above using Theorem 4.1(ii) shows that

$$
\lim _{k \rightarrow \infty} S_{\beta}(k)=E_{\beta}^{2} / 2
$$

so, by part (i) of this lemma

$$
\lim _{k \rightarrow \infty} \lambda_{k}^{2 \alpha+1} \int_{\pi / 2}^{\pi} \varphi_{k}^{2}(s) \rho^{2}(s) d s=\lim _{k \rightarrow \infty}\left[\lambda_{k}^{-\alpha-\beta} \varphi_{k}(\pi)\right]^{2} S_{\beta}(k)=E_{\alpha}^{2} / 2
$$

and part (iii) follows since

$$
\lambda_{k}^{2 \alpha+1} \int_{0}^{\pi} \varphi_{k}^{2}(s) \rho^{2}(s) d s=S_{\alpha}(k)+\left[\lambda_{k}^{\alpha-\beta} \varphi_{k}(\pi)\right]^{2} S_{\beta}(k)
$$
1 .

Finally part (iv) is a consequence of (4.1) and (4.2) and the fact that $\mathscr{J}_{\alpha}(0)=$

4.4 Theorem. Haar measure on $(H, *)$ is absolutely continuous with respect to Lebesgue measure and the normalized Haar measure is given by $d m(t)=$ $\rho^{2}(t) d t$.

Proof. Let $f \in C^{\infty}(H)$, then $u(s, t)=T^{s} f(t)$ satisfies (3.4) so that if $U(s)=$ $\int_{0}^{\pi} u(s, t) \rho^{2}(t) d t$, then (3.4) together with the Fundamental Theorem of Calculus yield

$$
\begin{aligned}
\left(\rho^{2} U^{\prime}\right)^{\prime}(s) & =\rho^{2}(s) \int_{0}^{\pi} \frac{\partial}{\partial t}\left[\rho^{2}(t) u_{t}(s, t)\right] d t \\
& =\rho^{2}(s)\left[\lim _{t \rightarrow \pi-} \rho^{2}(t) u_{t}(s, t)-\lim _{t \rightarrow 0+} \rho^{2}(t) u_{t}(s, t)\right] .
\end{aligned}
$$

Lemma 3.1 guarantees the limits to be zero because $f \in C^{\infty}(H)$ implies that $u_{t}(s, t)$ is bounded. 
Thus $\rho^{2}(s) U^{\prime}(s)=\lim _{s \rightarrow 0+} \rho^{2}(s) U^{\prime}(s)=0$. Hence $U$ is constant, so for $f \in C^{\infty}(H)$

$$
\int_{0}^{\pi} T^{s} f(t) \rho^{2}(t) d t=\int_{0}^{\pi} f(t) \rho^{2}(t) d t
$$

which yields the theorem since $C^{\infty}(H)$ is uniformly dense in $C(H)$.

4.5 Theorem. (i) $\mathrm{H}^{\wedge}$ is a complete set of eigenfunctions for the problem

$$
\left(\rho^{2} y^{\prime}\right)^{\prime}+\mu \rho^{2} y=0, \quad y^{\prime}(0)=y^{\prime}(\pi)=0 .
$$

(ii) $\varphi_{k}$ has exactly $k$ zeros interior to $H$.

Proof. This follows from Corollary 4.2, Lemma 3.3, and Theorem 4.4 since $H^{\curlywedge}$ is a complete orthogonal family for $L^{2}(d m)=L^{2}\left(\rho^{2}(t) d t\right)$.

It is now possible to obtain some precise estimates for $\lambda_{k}, \varphi_{k}$, and $h_{k}$.

4.6 Theorem. (i) $\lim _{k \rightarrow \infty} k^{-1} \lambda_{k}=1$.

(ii) $\varphi_{k}(\pi)=(-1)^{k} k^{\beta-\alpha}[E+o(1)]$.

(iii) $h_{k}^{-1 / 2}=\left\|\varphi_{k}\right\|_{2}=k^{-\alpha-1 / 2}\left[E_{\alpha}+o(1)\right]$.

(iv) There exists $K \geq 0$ such that $\varphi_{k}(t) \geq \frac{1}{3}$ if $k t \leq K$.

Proof. Part (i) is a consequence of Theorem 4.5(i) and Lemma 3.4. Parts (ii)(iv) (except for the alternating sign in part (ii)) are obtained by applying part (i) to parts (i), (iii), and (iv) of Theorem 4.3. The alternating sign in (ii) is due to the fact that, $\varphi_{k}(0)=1, \varphi_{k}$ has $k$ zeros interior to $H$ and $\varphi_{k}$ changes sign at each zero since it is a nontrivial solution of a second order differential equation which is normal in the interior of $H$.

The following result is a desirable property that is not known to hold in all compact commutative hypergroups; indeed Dunkl [8] is forced to include a rather strong hypothesis to obtain a similar result $\left[8\right.$, p. 340]. ${ }^{2}$

4.7 Theorem. The span of $H^{\wedge}$ is uniformly dense in $C(H)$.

Proof. First note that equation (3.2) and the differentiability of $(H, *)$ imply that $B \in C^{\infty}(0, \pi)$ so that $\rho \in C^{\infty}(0, \pi)$ also.

Let $C_{c}^{\infty}(0, \pi)$ be the space of functions with compact support in $(0, \pi)$, and let $f \in C_{c}^{\infty}(0, \pi)$. Then (3.4) and two partial integrations yield

$$
\begin{aligned}
f_{k}^{\widehat{\gamma}} & =\int_{0}^{\pi} f\left(\rho^{2} \varphi_{k}\right) d t=-\lambda_{k}^{-2} \int_{0}^{\pi} f\left(\rho^{2} \varphi_{k}^{\prime}\right)^{\prime} d t \\
& =-\lambda_{k}^{-2} \int_{0}^{\pi} \varphi_{k}\left(\rho^{2} f^{\prime}\right)^{\prime} d t=-\lambda_{k}^{-2} F_{k}^{-}
\end{aligned}
$$

where $F=\rho^{-2}\left(\rho^{2} f^{\prime}\right)^{\prime} \in C_{c}^{\infty}(0, \pi)$ so we can show inductively that $f_{k}=$ $O\left(\lambda_{k}^{-2 p}\right),(p=1,2, \ldots)$. Thus Theorem 4.6(iii) implies $\sum_{k=0}^{\infty} f_{k}^{\wedge} h_{k} \varphi_{k}$ converges uniformly to $f$, so $f$ is in the uniform closure of the span of $H^{-}$.

\footnotetext{
${ }^{2}$ See also K. Ross, Centers of hypergroups, Trans. Amer. Math. Soc. 243 (1978), 251-269.
} 
If $f \in C(H)$ it is possible to choose $a$ and $b$ so that $g=f+a \varphi_{0}+b \varphi_{1}$ satisfies $g(0)=g(\pi)=0$, so $g$ can be uniformly approximated by functions in $C_{c}^{\infty}(0, \pi)$, and hence so can $f$.

Proof of Theorem 3.6. Let $\rho$ be obtained from $(H, *)$ as in (3.3), then from Theorem 4.5 it follows that $H^{-}$constitutes a complete set of eigenfunctions for the Sturm-Liouville problem consisting of $\left(\rho^{2} y^{\prime}\right)^{\prime}+\lambda^{2} y=0$ and a boundary condition of the form given by (3.12). On the other hand, (3.11) and (3.12) imply that $H^{-}$is an orthogonal family for $L^{2}(w(t) d t)$. Thus, since $\varphi_{0}=1$,

$$
\int_{0}^{\pi} \varphi_{k}(t) \rho^{2}(t) d t=\int_{0}^{\pi} \varphi_{k}(t) w(t) d t=0 \quad(k=1,2, \ldots)
$$

so $(H, *)$ is of Jacobi type $(\alpha, \beta)$ by Theorem 4.7 .

4.8 The case $\alpha<0$ or $\beta<0$. The problems that arise in this case are due to the fact that if $\alpha<0$ (resp. $\beta<0$ ) then equation (4.10) has two linearly independent solutions which are bounded in $(0, \pi / 2)(\mathrm{resp} .(\pi / 2, \pi))$. This is easily resolved at 0 because the boundary condition in (3.2) implies $\varphi_{k}^{\prime}(0)=0$. When $\beta<0$ the difficulties at $\pi$ are eliminated by the following boundary condition:

$$
C \varphi_{k}(\pi)+D_{k}=0,
$$

where $D_{k}=\lim _{s \rightarrow \pi-} \rho^{2}(s) \varphi_{k}^{\prime}(s)$. Equation (4.11) ensures the orthogonality of $\left\{\varphi_{k}\right\}$ in $L^{2}\left(\rho^{2}(t) d t\right)$. This would certainly be the case if $\varphi_{k}^{\prime}(\pi)=0$ which would be the result if the boundary condition $u_{s}(\pi, t)=0$ is added to (3.2). The same condition would hold if $(H, *)$ is symmetric in the sense that $T^{\pi-s} f(\pi-t)=T^{s} f(t)$. Nevertheless, the results in the previous section still apply with the modifications discussed below.

Theorem 4.1(i) is valid for all $\alpha, \beta>-\frac{1}{2}$ because the condition $\varphi_{k}^{\prime}(0)=0$ forces $B_{k}=0$ in equation (4.5). When $-\frac{1}{2}<\alpha<0$ (4.7) can be replaced by the bounds:

$$
\left|H_{k}(s, t)\right| \leq \begin{cases}C(t / s)^{2 \alpha} & \left(0 \leq t \leq s \leq \lambda_{k}^{-1}\right), \\ C\left(\lambda_{k} s\right)^{-\alpha-1 / 2}\left(\lambda_{k} t\right)^{2 \alpha} & \left(0 \leq t \leq \lambda_{k}^{-1} \leq s\right), \\ C(t / s)^{\alpha+1 / 2}\left(\lambda_{k} t\right)^{-1} & \left(0 \leq \lambda_{k}^{-1} \leq t \leq s\right) .\end{cases}
$$

The rest of the analysis in the proof of part (i) remains valid. For part (ii) a similar, but necessarily more complicated result holds with (4.4) replaced by

$$
\begin{aligned}
\varphi_{k}(s)= & \varphi_{k}(\pi)\left[a_{\beta}(\pi-s)^{\beta+1 / 2} / \rho(s)\right] \mathscr{F}_{\beta}\left(\lambda_{k}(\pi-s)\right) \\
& -D_{k}(\pi-s)\left[2 \beta a_{\beta}(\pi-s)^{\beta+1 / 2} \rho(s)\right]^{-1} \mathscr{J}_{-\beta}\left(\lambda_{k}(\pi-s)\right)+M_{k}(s) .
\end{aligned}
$$

Finally if $\alpha=-\frac{1}{2}$ it follows from [20, Lemma 1.7(ii)] that

$$
\varphi_{k}(s)=\frac{a_{-1 / 2}}{\rho(s)} \cos \left(\lambda_{k} s\right)+I_{k}(s)
$$


with $\left|I_{k}(s)\right| \leq C \lambda_{k}^{-1}$, and if $\beta=-\frac{1}{2}$

$$
\varphi_{k}(s)=\varphi_{k}(\pi) \frac{a_{-1 / 2}}{\rho(s)} \cos \left(\lambda_{k}(\pi-s)\right)+M_{k}(s)
$$

with $\left|M_{k}(s)\right| \leq C \lambda_{k}^{-1}$

These changes have negligible effect on the behavior of $\varphi_{k}(x), 0<x<$ $\pi$, for large values of $k$ because if $\beta<0, \mathscr{J}_{\beta}\left(\lambda_{k}(\pi-s)\right)$ oscillates with an amplitude $O\left(\lambda_{k}^{-\beta-1 / 2}\right)$ while $\mathscr{J}_{-\beta}\left(\lambda_{k}(\pi-s)\right)$ has an amplitude which is at least a constant times $\lambda_{k}^{\beta-1 / 2}$. Thus Theorem 4.3 stands unchanged. Theorem 4.4 remains valid since its proof does not depend on boundary conditions at all.

For $\beta>-1 / 2$ the added boundary condition (4.11) ensures that the orthogonality of $\left\{\varphi_{k}\right\}$ in $L^{2}\left(\rho^{2}(t) d t\right)$, so the conclusions of Theorems 4.5 and 4.6 are still true if the boundary condition at $\pi$ is included in the hypotheses.

When $\alpha \geq \beta=-1 / 2$, the proof of Theorem 4.4 is still valid with the added hypothesis that $\lim _{t \rightarrow \pi-} \partial T^{t} f(s) / \partial t=0$ whenever $f$ belongs to some dense subset of $C(H)$. The same is true for Theorems 3.6 and 4.5-4.7.

4.9 Remarks. The boundedness condition which was added to differentiability at the beginning of $\S 2$ is used only in the proof of Theorem 4.4; consequently, it is possible to replace this with any of the following conditions:

C1. The span of $H^{-}$is uniformly dense in $C(H)$.

C2. $(H, *)$ is a symmetric hypergroup.

C3. For all $f$ belonging to a uniformly dense subset of $C(H), T^{s} f(t)$ has bounded second order derivatives on the interior of $H \times H$.

C4. The following three relations hold for all $f$ belonging to a uniformly dense subset of $C(H)$ :

$$
\begin{gathered}
\frac{\partial}{\partial s} \rho^{2}(s) \frac{\partial}{\partial s} \int_{0}^{\pi} T^{s} f(t) d t=\int_{0}^{\pi} \frac{\partial}{\partial s} \rho^{2}(s) \frac{\partial}{\partial s} T^{s} f(t) d t, \\
\lim _{s \rightarrow 0+} \rho^{2}(s) \frac{\partial}{\partial s} \int_{0}^{\pi} T^{s} f(t) d t=0 \\
\lim _{s \rightarrow \pi-} \rho^{2}(s) \frac{\partial}{\partial s} T^{s} f(t)=0 .
\end{gathered}
$$

Condition C4 is an obvious minimal condition for the proof of Theorem 4.4 to be valid as given.

Condition C3 can be used to establish equations (4.12)-(4.14) so C3 is stronger than $\mathrm{C} 4$.

To see that $\mathrm{C} 2$ suffices, assume that $(H, *)$ is a symmetric hypergroup and that $f \in C_{c}^{\infty}(0, \pi)$. Let $u(s, t)=T^{s} f(t)$. Then for each fixed $s$ which is sufficiently small $u(s, t) \in C_{c}^{\infty}(0, \pi)$. Also $\lim _{s \rightarrow 0+} u_{s s}(s, t)=L f(t)$ where $L$ is a differential operator, thus as $s$ approaches $0, u_{s s}(s, t)$ converges uniformly to a continuous function. Condition C2 implies $u(\pi-s, \pi-t)=u(s, t)=$ $u(t, s)$ for every $s, t \in H$. Thus $\mathrm{C} 3$ holds. 
Since $\varphi_{0}=1$, it is clear that equation (4.9) holds when $f=\varphi_{k}, k=$ $0,1,2, \ldots$, thus $(4.9)$ holds for $f$ belonging to the span of $H^{\wedge}$. If $\mathrm{C} 1$ holds, this yields (4.9) for all $f \in C(H)$.

\section{EXAMPLes (continued)}

A. $(K, \circ ; \alpha, \beta)$ is a hypergroup of Jacobi type $(\alpha, \beta)$ so the results of the previous sections generalize properties of the hypergroup $(K, \circ ; \alpha, \beta)$ the notations of that example will continue in this section.

Condition $\mathrm{C} 1$ holds by the Weierstrass Polynomial Approximation Theorem since for each $k, R_{k}$ is a polynomial of degree $k$.

An explicit formula for $R_{1}(\cos \theta)$ can be obtained from [19, equation (4.3.2)] so that

$$
\begin{gathered}
\lim _{\varphi \rightarrow 0+} a_{2}^{-1} \sin \varphi A_{1}(\varphi)=\alpha+\frac{1}{2}, \\
\lim _{\varphi \rightarrow \pi-} a_{2}^{-1} \sin \varphi A_{1}(\varphi)=-\left(\beta+\frac{1}{2}\right) .
\end{gathered}
$$

which is equivalent to (3.1), and it is easily derived from equations (1.7) and (1.8) that the parameters in Lemma 3.1(i) are $a_{\alpha}=2^{-\alpha-1 / 2} B$ and $a_{\beta}=$ $2^{-\beta-1 / 2} B$.

Let $\varphi_{k}(\theta)=R_{k}(\cos \theta)$. If $y(\theta)=\rho(\theta) \varphi_{k}(\theta)$ then $y$ is a solution of

$$
y^{\prime \prime}+\left(\lambda_{k}^{2}-q\right) y=0
$$

where $\lambda_{k}=k+(\alpha+\beta+1) / 2[19$, equation (4.24.2)] and

$$
q(\theta)=\frac{\alpha^{2}-1 / 4}{4 \sin ^{2} \theta / 2}+\frac{\beta^{2}-1 / 4}{4 \cos ^{2} \theta / 2}
$$

which is in agreement with Lemma 3.2 and Theorem 4.6(i). Also

$$
q(\theta)=\frac{\alpha^{2}-1 / 4}{\theta^{2}}+\frac{\beta^{2}-1 / 4}{(\pi-\theta)^{2}}+Q(\theta)
$$

with $Q$ belonging to $C(K)$ which is actually stronger than Lemma 3.1(ii).

It is a simple exercise to show that $y=\varphi_{k}$ solves (3.11) with

$$
w(\theta)=\left(\sin \frac{\theta}{2}\right)^{2 \alpha+1}\left(\cos \frac{\theta}{2}\right)^{2 \beta+1}
$$

which shows that Theorem 3.6 provides a much simpler way of determining that $(K, \circ ; \alpha, \beta)$ is a hypergroup of Jacobi type $(\alpha, \beta)$ than the direct calculation of $M_{K, 1}$ and $M_{K, 2}$.

The Hilb-type estimate [19, Theorem 8.21.12] yields (4.1) with the smaller error terms:

$$
\begin{array}{ll}
O\left(\lambda_{k}^{-2}\right) & \left(0 \leq \theta \leq \lambda_{k}^{-1}\right), \\
O\left[\lambda_{k}^{-1}\left(\lambda_{k} \theta\right)^{-\alpha-1 / 2}\right] & \left(\lambda_{k} \leq \theta \leq \pi-\varepsilon\right),
\end{array}
$$


in place of those in equations (4.2) and (4.3). These better estimates are smaller because in this example $Q(\theta)$ is bounded, whereas in a general Jacobi type hypergroup it is only assumed that $\sin \theta Q(\theta)$ is bounded.

From $[19,4.1 .4]$ it follows that

$$
\varphi_{k}(\pi)=(-1)^{k}\left(\begin{array}{c}
k+\alpha \\
k
\end{array}\right)^{-1}\left(\begin{array}{c}
k+\beta \\
k
\end{array}\right)
$$

and Stirling's formula yields

$$
\varphi_{k}(\pi)=(-1)^{k}\left(\frac{\Gamma(\alpha+1)}{\Gamma(\beta+1)}+o(1)\right) k^{\beta-\alpha}
$$

which agrees with Theorem 4.7(ii).

Equation (1.9) yields

$$
\left\|\varphi_{k}\right\|_{2}^{2}=\left(\begin{array}{c}
k+\alpha \\
k
\end{array}\right)^{-1}\left(\begin{array}{c}
k+\beta \\
k
\end{array}\right)\left(\begin{array}{c}
k+\alpha+\beta \\
k
\end{array}\right)^{-1} \frac{\alpha+\beta+1}{2 k+\alpha+\beta+1}
$$

which is seen to agree with Theorem 4.6(iii) by means of Stirling's formula.

B. The Fourier-Bessel basis functions of Example B are eigenfunctions of the operator $L y=y^{\prime \prime}+2 B y^{\prime}$ where $B(x)=\left(\alpha+\frac{1}{2}\right) / x$. The characters of the associated hypergroup are $\varphi_{k}(x)=\widetilde{J}_{k}^{\alpha}(x)$ and they satisfy $\varphi_{k}^{\prime}(0)=\varphi_{k}^{\prime}(\pi)=0$. This last equality can be established with the help of the recurrence relations for Bessel functions in $[21, \S 3.2]$. Condition $\mathrm{Cl}$ obtains since the span of $\mathrm{H}^{-}$is uniformly dense in $C_{c}(0,1)$, and hence in $C(H)$ (see $\left.[21, \S 18.53]\right)$. It follows from Theorem 3.6 that the resulting hypergroups are of Jacobi type $\left(\alpha-\frac{1}{2}\right)$ when $\alpha \in\left\{\frac{1}{2}, \frac{3}{2}, \frac{5}{2}\right\}$. (In this example the interval is $[0,1]$ instead of $[0, \pi]$ ).

C. A similar examination of the perturbed ultraspherical case with a sufficiently smooth perturbation $q$ satisfying the conditions previously specified shows the resulting hypergroup to be symmetric (condition $\mathrm{C} 1$ ) and of Jacobi type $(\alpha, \alpha)$ with the normalized eigenfunctions as characters. The characters satisfy the boundary condition $\varphi_{k}^{\prime}(0)=\varphi_{k}^{\prime}(\pi)=0$ because of symmetry.

\section{REFERENCES}

1. E. A. Coddington and N. Levinson, Theory of ordinary differential equations, McGraw-Hill, New York, 1955.

2. W. C. Connett, C. Markett, and A. L. Schwartz, Convolution and hypergroup structures associated with a class of Sturm-Liouville eigenfunction systems, (preprint).

3. W. C. Connett and A. L. Schwartz, A multiplier theorem for Jacobi expansions, Studia Math. 52 (1975), 243-261.

4. ${ }_{1-92}$, The theory of ultraspherical multipliers, Mem. Amer. Math. Soc., No. 183, 1977, pp.

5. __, The Littlewood-Paley theory for Jacobi expansions, Trans. Amer. Math. Soc. 251 (1979), 219-234.

6. __ The harmonic machinery for eigenfunction expansions, Proc. Sympos. Pure Math., vol. 35, Amer. Math. Soc., Providence, R. I., 1979, pp. 429-434.

7. __ Convolution structures for eigenfunction expansions arising from regular SturmLiouville problems, Anniversary Volume on Functional Analysis and Approximation, (P. L. Butzer, R. L. Stens, and B. Sz.-Nagy, Eds.), Birkhäuser, Basel, 1984, pp. 437-447. 
8. C. F. Dunkl, The measure algebra of a locally compact hypergroup, Trans. Amer. Math. Soc. 179 (1973), 331-348.

9. G. Gasper, Banach algebras for Jacobi series and positivity of a kernel, Ann. of Math. 95 (1972), 261-280.

10. J. E. Gilbert, Maximal theorems for some orthogonal series. II, J. Math. Anal. Appl. 31 (1970), 349-368.

11. H. Heyer, Probability theory on hypergroups: a survey, Probability Measures on Groups VII, Proceedings Oberwolfach 1983, Lecture Notes in Math., vol. 1064, Springer, Berlin.

12. R. I. Jewett, Spaces with an abstract convolution of measures, Adv. in Math. 18 (1975), $1-101$.

13. B. M. Levitan, Generalized translation operators, Israel Program for Scientific Translations, Jerusalem, 1964.

14. G. L. Litvinov, Hypergroups and hypergroups algebras, J. Soviet Math. 38 (1987), 17341761.

15. C. Markett, Product formulas and convolution structure for Fourier-Bessel series, Constr. Approx. 5 (1989), 383-404.

16. E. Michael, Topologies on spaces of subsets, Trans. Amer. Math. Soc. 71 (1951), 152-182.

17. A. L. Schwartz, Classification of one-dimensional hypergroups, Proc. Amer. Math. Soc. 103 (1988), 1073-1081.

18. R. Spector, Measures invariantes sur les hypergroupes, Trans. Amer. Math. Soc. 239 (1978), 147-165.

19. G. Szegö, Orthogonal polynomials, Amer. Math. Soc. Colloq. Publ., vol. 23, Amer. Math. Soc., Providence, R. I., 1939.

20. E. C. Titchmarsh, Eigenfunction expansions associated with second-order differential equations, Oxford Univ. Press, London, 1969.

21. G. Watson, $A$ Treatise on the theory of Bessel functions, Cambridge Univ. Press, Cambridge, 1966.

Department of Mathematics, University of Missouri, St. Louis, Missouri 63121-4499 